\section{A rare case of polypoid primary anorectal melanoma with subsequent giant stomach metastasis: A gastrointestinal involvement of both primary and metastatic mucosal melanoma}

\author{
Vincenzo De Giorgi, ${ }^{1}$ Federica Scarfi, ${ }^{1}$ \\ Carlo Boselli, ${ }^{2}$ Giacomo Sacchetti, ${ }^{2}$ \\ Nicola Natalizi, ${ }^{2}$ Danilo Castellani, ${ }^{3}$ \\ Piero Covarelli ${ }^{2}$ \\ ${ }^{1}$ Department of Dermatology, University \\ of Florence; ${ }^{2}$ Department of Surgery, \\ University of Perugia; ${ }^{3}$ Gastroenterology \\ and Digestive Endoscopy Unit, Perugia \\ General Hospital, Italy
}

\begin{abstract}
Melanoma can involve the gastrointestinal apparatus as both primary and metastatic lesions. Primary anorectal mucosal melanoma (ARMM) and metastatic gastric melanoma are rare entities and usually resulted in a poor prognosis. We presented a case of a 61-year-old man who after the complete excision of an ARMM developed a gastric metastasis after almost three years form the complete tumour excision. Upon esophagogastroduodenoscopy, a giant ulcered mass resulted in melanoma metastasis. The patient underwent a near-total gastrectomy. After five months of follow-up, the patient is disease-free. The incidence of ARMMs is increasing, highlighting the necessity of new prevention and treatment strategies in order to achieve a better prognosis for these patients. There are no known risk factor for ARMMs but surgery, together with the combination of anti-CTLA-4 and anti-PD-1 antibodies, are promising therapeutic options. Early and aggressive treatments are required, together with a strict multidisciplinary approach.
\end{abstract}

\section{Introduction}

Uncommonly, the gastrointestinal tract can be both the primary or the metastatic site of melanoma disease. Herein, we report the case of a primary anorectal mucosal melanoma (ARMM) treated with wide local excision that developed a giant amelanotic gastric metastasis after three years of follow-up.

\section{Case Report}

A 61-year-old male patient was referred to the Surgery Unit of the University of Perugia, for a complete excision of an ARMM diagnosed upon biopsy. The patient had multiple severe comorbidities: chronic ischemic heart disease, hypertension, hyperuricemia, class 1 obesity (BMI 34.6 $\mathrm{kg} / \mathrm{m}^{2}$ ), type 2 diabetes complicated by neuropathy, micro-and macroangiopathy, chronic renal failure and he had the amputation of the left leg in 2011 due to critical ischemia. In November 2017, the patient underwent an incisional excision with the partial removal of a bleeding anal mass. The histopathological exam revealed the presence of an ulcerated ipomelanotic polypoid malignant melanoma, extended to the implant base. Immunohistochemical analysis of the specimen showed positivity for S100, MART-1 and HMB-45 markers +, and negativity for PANCK. The patient was then referred to our Surgery Unit; upon clinical examination, a large anal polyp partially pigmented mass protruding from anus was visible. No palpable axillary and inguinal lymph nodes were present. In December 2017, we performed a wide local excision of the anal area (Figure 1) coupled with a right groin sentinel node biopsy. This latter resulted in the presence of clinical occult micrometastasis in one lymph node. A fullbody computed tomography scans, performed after surgery, excluded the presence of other melanoma metastasis. Upon molecular analysis, no mutation was found in BRAF, NRAS and cKIT genes. The stage of the disease was IIIC (pT4bN1aM0), according to the $8^{\text {th }}$ AJCC melanoma staging.

The multidisciplinary tumour board decided not to perform the inguinal completion lymphadenectomy, considering the patient's general condition. The subject was then addressed to oncologists care. He refused any medical treatment, including immunotherapy and he performed threemonths follow-up visits that resulted negative for disease progression till his last oncologic control on February 2020.

In June 2020 the patient was hospitalized for severe acute anaemia (HB 6.5 $\mathrm{g} / \mathrm{dL}$ ), treated with GRC transfusion and investigated by esophagogastroduodenoscopy (EGDS) with evidence of erosive antral gastritis and extensive ulcer of the gastric body (Figure 2A). Multiple biopsies specimens of the gastric mucosa were taken and revealed metastatic melanoma with the following immune-histochemical profile: PANCK-, S-100 +, SOX10 +, MART1 +, HMB45+. A total body positron emission tomography-computed tomogra-
Correspondence: Vincenzo De Giorgi, Department of Dermatology, University of Florence, Via Michelangelo 41, 50124 Firenze, Italy.

Tel: +39.055.6939632 - Fax +39.055.6939632.

E-mail: vincenzo.degiorgi@unifi.it

Key words: Primary anorectal melanoma, Gastric metastasis, Surgery, Endoscopy.

Contributions: VDG had full access to all of the data in the study and takes responsibility for the integrity of the data and the accuracy of the data analysis. Study concept and design: VDG, PC. Acquisition of data: PC, CB, GS, NN, DC. Analysis and interpretation of data: VDG, PC, FS. Drafting of the manuscript: VDG, FS. Critical revision of the manuscript for important intellectual content: VDG, FS. Study supervision: VDG.

Conflict of interest: The authors declare no potential conflict of interest.

Funding: None.

Ethical approval and informed consent: Received.

Availability of data and material: Data and materials are available by the authors.

Please cite this article as: De Giorgi V, Scarfi $F$, Boselli $C$, et al. A rare case of polypoid primary anorectal melanoma with subsequent giant stomach metastasis: A gastrointestinal involvement of both primary and metastatic mucosal melanoma. Dermatol Rep 2021;13:9092.

Received for publication: 31 January 2021.

Accepted for publication: 23 February 2021.

This work is licensed under a Creative Commons Attribution-NonCommercial 4.0 International License (CC BY-NC 4.0).

(C) Copyright: the Author(s), 2021

Licensee PAGEPress, Italy

Dermatology Reports 2021; 13:9092

doi:10.4081/dr.2021.9092

phy (PET-CT) scan was performed, which evidenced an SUV-elevated lesion at the large gastric curvature, without other signs of disease spreading. During the multidisciplinary oncologic meeting, the excision of the gastric mass was suggested in order to avoid possible massive bleeding during the treatment. At surgery, the lesion presented as 7 centimetres, exophytic and infiltrating amelanotic mass. A near-total gastrectomy was performed. The final histopathological diagnosis was: "full-thickness infiltration of the gastric wall of melano-epitheliomorphic cells, with upper and lower resection mar- 
gins free from proliferations neoplastic and immunohistochemical profile PANCK-, SOX10+, MART1+, HMB45+".

The postoperative course was uneventful, and the patient was dismissed seven days after surgery. After five-months follow-up, the patient is still disease-free.

\section{Discussion and conclusions}

Primary ARMM and metastatic gastric melanoma are both rare entities. Because of their aspecific and uncommon presentation, they are found in advanced melanoma stages, and for this reason, they are associated with a poor prognosis. ${ }^{1,2}$ In the last years, the number of ARMMs is increasing, pointing out questions about its pathogenesis and the possible strategies of prevention. ${ }^{3}$ A recent meta-analysis assesses that the standard of care is represented by wide local excision (WLE) which should be preferred to abdominoperineal resection (APR) due to the equal outcome regarding the overall survival, the quicker rehabilitation and the possible preservation of the sphincter. ${ }^{4}$ For this reason, we performed WLE in our patient, who already had multiple known comorbidities.

Although the response to adjuvant medical treatments is lower in advanced ARMMs compared to advanced primary cutaneous melanomas, checkpoint inhibitors, especially a combination of antiCTLA-4 and anti-PD-1 antibodies, seem to be a promising therapeutic option when associated with local agents. ${ }^{5}$
Regarding the metastatic involvement of the GI tract, the most common cancers implicated are melanoma, lung and breast cancer; in particular, the stomach is compromised in melanoma in about $20 \%$ of cases. ${ }^{6}$ In the majority of events, the endo- scopic presentation consists of a unique, non-pigmented, ulcerated lesion in the gastric body, where multiple biopsies are diagnostic; as it happened in our case. ${ }^{7}$ Another manifestation consisted of pigmented nonulcerated lesions. Recently also endoscopic

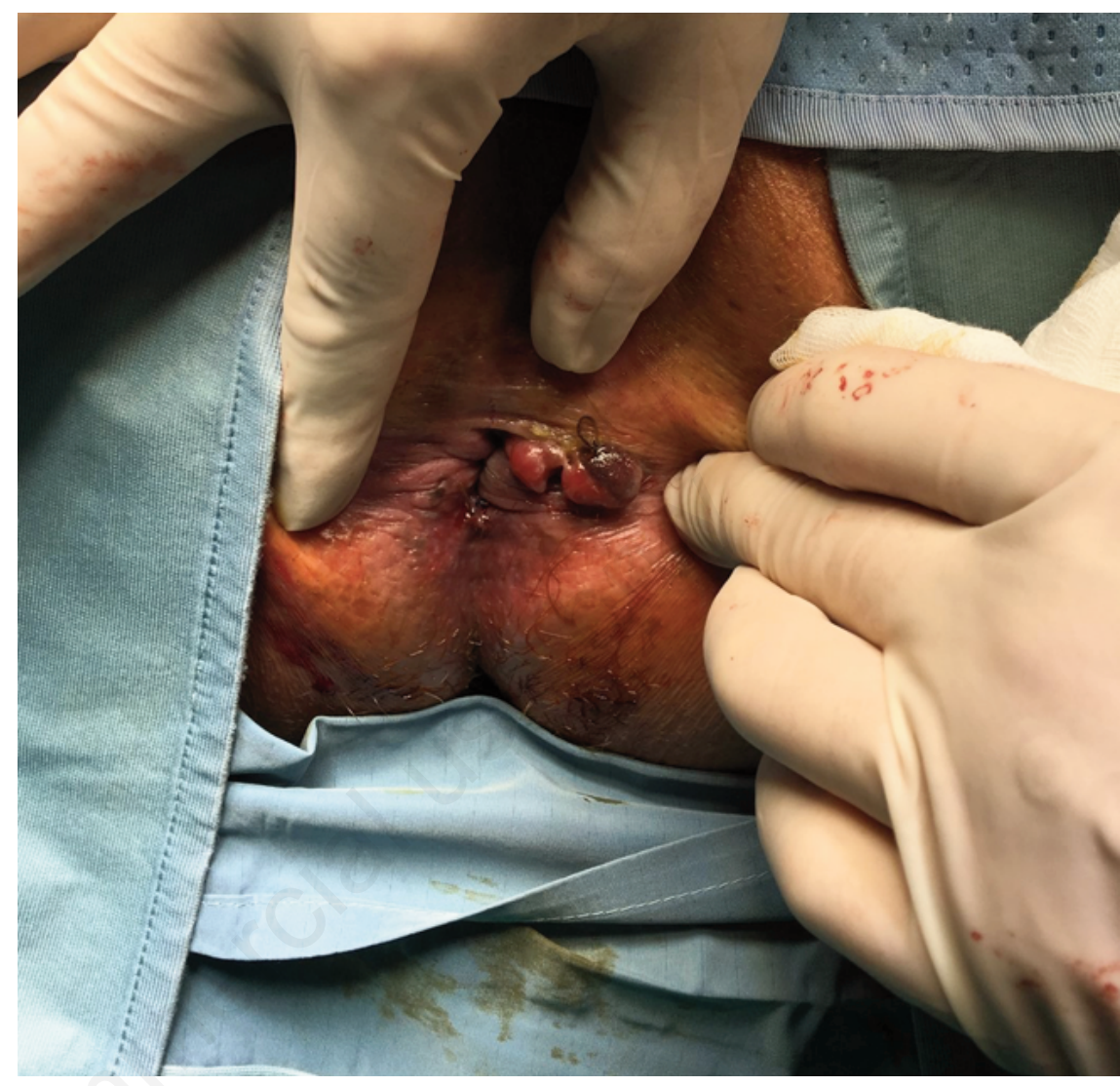

Figure 1. Preoperative clinical imagine: the large partially pigmented polyp mass of the primary mucosal melanoma.
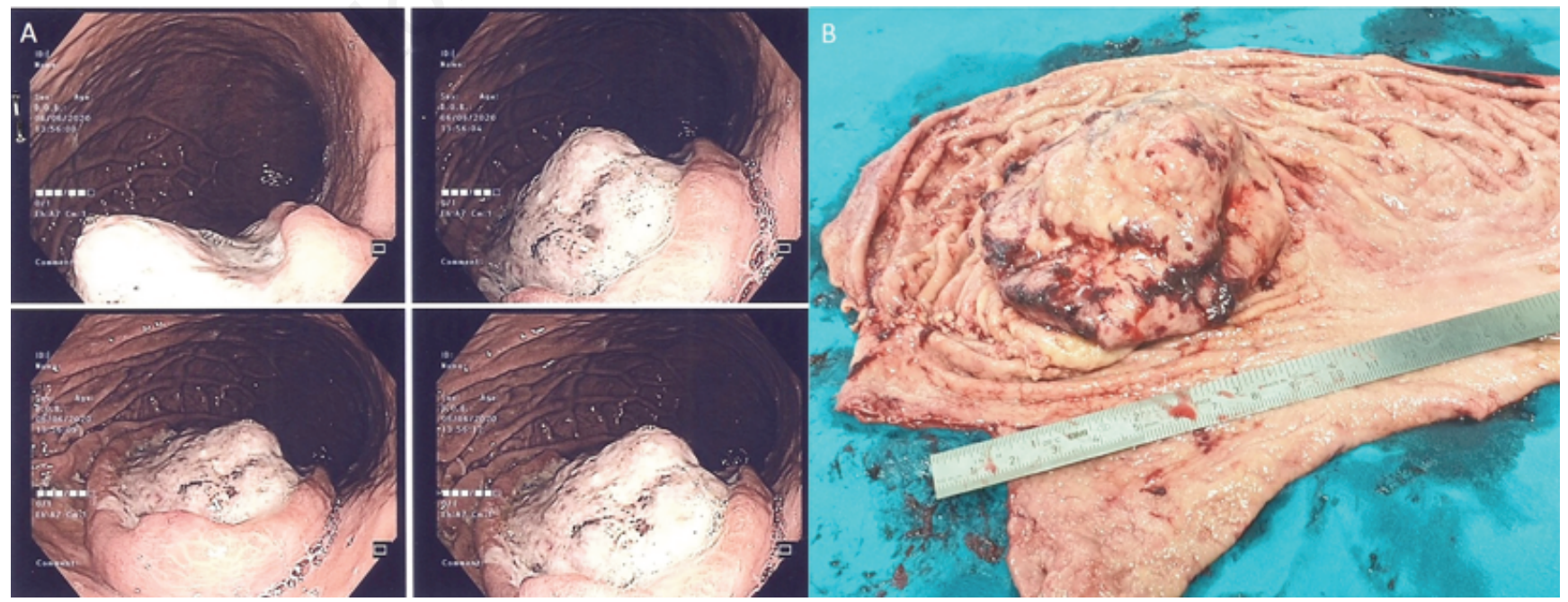

Figure 2. Endoscopic images of the extensive no pigmented ulcerated mass of the gastric body (A). Surgical specimen consisting of an almost seven centimeter pinkish metastatic melanoma mass $(B)$.primary mucosal melanoma. 
full-thickness resection was demonstrated feasible for gastric lesion; this option was not practicable in our patient due to the huge dimension of the gastric metastase. ${ }^{8}$

This is a rare case report of an ARMM with gastrointestinal (GI) tract as the unique site of metastasis. The management of metastatic ARMM is still challenging; we maintain that an aggressive treatment based on a multidisciplinary approach is crucial.

\section{References}

1. Yeung HM, Gupta B, Kamat B. A Rare Case of Primary Anorectal Melanoma and a Review of the Current Landscape of Therapy. J Community Hosp Intern
Med Perspect 2020;10:371-6.

2. Liang KV, Sanderson SO, Nowakowski GS, Arora AS. Metastatic malignant melanoma of the gastrointestinal tract. Mayo Clin Proc 2006;81:511-6.

3. Taylor JP, Stem M, Yu D, et al. Treatment Strategies and Survival Trends for Anorectal Melanoma: Is it Time for a Change?. World J Surg 2019;43:1809-19.

4. Smith HG, Glen J, Turnbull N, et al. Less is more: A systematic review and meta-analysis of the outcomes of radical versus conservative primary resection in anorectal melanoma. Eur $\mathrm{J}$ Cancer 2020;135:113-20.

5. Klemen ND, Wang M, Rubinstein JC, et al. survival after checkpoint inhibitors for metastatic acral, mucosal and uveal melanoma. $\mathrm{J}$ Immunother Cancer 2020;8:e000341. 1

6. Bahat G, Saka B, Colak Y, Tascioglu C, Gulluoglu M. Metastatic gastric melanoma: a challenging diagnosis. Tumori 2010;96:496-7.

7. Haendchen Bento L, Kazuyoshi Minata M, Pires Batista C, et al. Clinical and endoscopic aspects of metastases to the gastrointestinal tract. Endoscopy 2019;51:646-52.

8. Kratt T, Kueper M, Boesmueller H, et al. Endoscopic full-thickness resection of gastric metastasis from malignant melanoma by use of a novel over-thescope device. Gastrointest Endosc 2016;84:368. 